Biogeografía

\title{
Primer registro fósil del gasterópodo Cincinnatia (Hydrobiidae: Nymphophilinae) en México
}

\author{
First record of fossil gastropod Cincinnatia (Hydrobiidae: Nymphophilinae) in Mexico \\ Alexander Czaja, José Luis Estrada-Rodríguez*, Ulises Romero-Méndez, \\ Josué Raymundo Estrada-Arellano y Alberto González-Zamora \\ Facultad de Ciencias Biológicas, Universidad Juárez del Estado de Durango, Avenida Universidad s/n, Fraccionamiento Filadelfia, 35010 Gómez Palacio, \\ Durango, México \\ Recibido el 11 de enero de 2017; aceptado el 1 de junio de 2017 \\ Disponible en Internet el 23 de noviembre de 2017
}

\section{Resumen}

Se describe el primer registro en México de conchas fósiles del gasterópodo de aguas continentales del género Cincinnatia Pilsbry, 1891, encontrados en sedimentos fluviátiles del Pleistoceno tardío en el valle de Acatita, Coahuila. La morfología y el análisis discriminante de las conchas indican que el material fósil corresponde a Cincinnatia integra (Say, 1821), una especie reciente con una distribución disjunta entre la parte oriental de los EUA y sur de Canadá y un área pequeña en la cuenca del río Pánuco, San Luís Potosí, México. Los nuevos hallazgos marcan el límite más occidental de la distribución original del género Cincinnatia y sugieren una conexión entre ambas áreas norte-sur en la extensión original de $C$. integra, hoy separadas, según registros, por más de $700 \mathrm{~km}$, desde el Pleistoceno tardío hasta tiempos subrecientes. Considerando que el norte de México es hasta la fecha una región poco muestreada con referencia a los miembros de la familia Hydrobiidae, existe una alta probabilidad de hallazgos de poblaciones vivas de Cincinnatia en las cuencas altas de esta región así como en sitios acuáticos de la parte norte de los estados de Coahuila, Nuevo León y Tamaulipas.

(C) 2017 Universidad Nacional Autónoma de México, Instituto de Biología. Este es un artículo Open Access bajo la licencia CC BY-NC-ND (http://creativecommons.org/licenses/by-nc-nd/4.0/).

Palabras clave: Mollusca; Norte de México; Este de Estados Unidos de América

\begin{abstract}
The first record of fossil shells of the freshwater gastropod genus Cincinnatia Pilsbry, 1891 found in fluvial sediments of the late Pleistocene in the Acatita Valley, Coahuila, Mexico, is described. Morphology and discriminant analysis indicate that the fossil material correspond to Cincinnatia integra (Say, 1821), an extant species with a disjunct distribution between the eastern part of United States of America and southern Canada and a small area near Pánuco River, San Luis Potosí, Mexico. The new findings mark the westernmost limit of the original distribution of Cincinnatia and suggest a north-south connection between both areas in the original extension of $C$. integra, today separated according to the records by more than $700 \mathrm{~km}$, since the late Pleistocene to sub-recent times. Considering that northern Mexico to date is under-sampled for the Hydrobiidae, there is a high probability of finding live populations of Cincinnatia in this region and in other aquatic habitats in the northern part of the states of Coahuila, Nuevo León, and Tamaulipas.
\end{abstract}

(C) 2017 Universidad Nacional Autónoma de México, Instituto de Biología. This is an open access article under the CC BY-NC-ND license (http://creativecommons.org/licenses/by-nc-nd/4.0/).

Keywords: Mollusca; Northern Mexico; Eastern United States of America

\footnotetext{
* Autor para correspondencia.

Correo electrónico: drjlestrada @ujed.mx (J.L. Estrada-Rodríguez).

La revisión por pares es responsabilidad de la Universidad Nacional Autónoma de México.
} 


\section{Introducción}

Cincinnatia Pilsbry, 1891 es un género monotípico de gasterópodos de agua dulce de la familia Hydrobiidae, subfamilia Nymphophylinae, con su especie Cincinnatia integra (Say, 1821), conocida como «midland siltsnail». Hershler y Thompson (1996) mencionan que la especie es además de interés particular en la malacología del norte de América ya que fue descrita como uno de los primeros taxones de gasterópodos en 1821 (como Paludina integra Say, 1821). Los mismos autores reportan la compleja historia de la nomenclatura de $C$. integra e incluyen además una larga lista de sinónimos. Hoy $C$. integra está distribuida en el este de EUA y en pocos lugares en Canadá (Hershler y Thompson, 1996) (fig. 1). Recientemente fueron reportados los primeros hallazgos de poblaciones vivas de C. integra en México, cerca de la localidad Veinte de Noviembre, río La Ciénega, San Luis Potosí, aproximadamente 700 km al sur de la distribución principal (Hershler, Thompson y Liu, 2011). Esta gran disyunción en la distribución motivó a Hershler et al. (2011) a realizar un estudio morfológico y molecular de las nuevas poblaciones mexicanas con el objetivo de analizar la posibilidad de la existencia de una nueva especie del género Cincinnatia en México; sin embargo, sus análisis estadísticos de las diferencias morfológicas de las conchas, así como de la divergencia genética de tan solo 1.5-2.6\% entre secuencias parciales del gen mitocondrial citocromo oxidasa I de especímenes de diferentes poblaciones no permitió la distinción de una nueva línea filogenética independiente.

Hallazgos fósiles de conchas de Cincinnatia han sido reportados hasta la fecha solamente de depósitos interglaciales del Pleistoceno y Holoceno temprano en sitios de EUA y Canadá (La Rocque, 1970). La ocurrencia de conchas del género Cincinnatia en el Plioceno de Waucobi Lake Beds, Sierra Nevada, California (Oseguera, 2012) es controversial ya que las conchas presentadas por la autora como Paludina integra (p. 68, fig. 2) no señalan la apertura y pueden pertenecer a cualquier otro género de las familias Hydrobiidae o Cochliopidae.

Czaja, Palacios-Fest, Estrada-Rodríguez, Romero-Méndez y Alba-Ávila (2014) describieron una malacofauna del Pleistoceno tardío proveniente de dunas terrestres del valle de Acatita, Coahuila, México. Entre los restos preservados de esta fauna se encontraron conchas, determinadas entonces provisionalmente como Pyrgulopsis sp. Una revisión crítica de estas conchas y el análisis morfométrico realizado en el presente trabajo demostraron sin embargo, que estas no pertenecen al género Pyrgulopsis Call y Pilsbry, 1886 sino al género hermano Cincinnatia. Adicionalmente al material de Acatita, 2 ejemplares de Cincinnatia fueron encontrados en sedimentos subrecientes en el manantial El Cerrito, cerca de Viesca, Coahuila. Los manantiales de Viesca se secaron en los años 50 del siglo XX (Czaja, EstradaRodríguez y Romero-Méndez, 2015), así que la especie tuvo aparentemente presencia (sin registros) en la región hasta tiempos recientes. Considerando que el norte de México hasta la fecha es una región poco muestreada referente a los miembros de la familia Hydrobiidae, existe una alta probabilidad de hallazgos de poblaciones vivas de esta interesante especie sobre todo en las cuencas altas en el norte de México.

El objetivo del presente trabajo es la descripción y análisis morfométrico de las conchas fósiles del género Cincinnatia encontradas por primera vez en México y su comparación con conchas de poblaciones recientes de Texas, Estados Unidos y del río La Ciénega, San Luis Potosí, México. La presente investigación forma parte del proyecto sobre la sistemática, ecología y biogeografía de moluscos de ecosistemas terrestres y acuícolas del norte de México iniciado por los autores en el 2013 (Czaja y Estrada-Rodríguez, 2015; Czaja, Estrada-Rodríguez y Romero-Méndez, 2014; Czaja, Palacios-Fest et al., 2014).

\section{Materiales y métodos}

Las poblaciones de conchas de $C$. integra consideradas en el presente trabajo fueron encontradas en 2 sitios en el suroeste de Coahuila, México (fig. 1). El material del primer sitio proviene de la parte sur del valle de Acatita y fue recolectado directamente de un corte de aproximadamente $2 \mathrm{~m}$ de espesor con sedimentos fluviátiles compuestos por gravas y arenas ( $26^{\circ} 16^{\prime} 53.77^{\prime}$ ' N, $103^{\circ} 04^{\prime} 25.96$ ” O). Sedimentos fluviátiles similares de varios sitios vecinos fueron fechados por Butzer et al. (2008) como Pleistoceno tardío ( 15,000 AP, método ${ }^{14} \mathrm{C}$, detalles sobre el sitio véase Czaja et al., 2014; Czaja y EstradaRodríguez, 2015). Dos ejemplares adicionales de $C$. integra fueron encontrados en la salida del manantial El Cerrito (fig. 1) ( $25^{\circ} 20^{\prime} 23^{\prime \prime}$ N, $102^{\circ} 54^{\prime} 29^{\prime \prime}$ O), cerca del poblado Viesca, Coahuila y provienen de sedimentos subrecientes. El manantial El Cerrito se encuentra aproximadamente $80 \mathrm{~km}$ al sur del valle de Acatita.

Las mediciones de las variables morfométricas de las conchas y la documentación fotográfica se realizaron con un microscopio estereoscópico Zeiss AxioCam ERc5s. Las vueltas del material estudiado fueron contadas según el método de Pilsbry (1939), incluyendo en la medición la vuelta de la protoconcha. Para el análisis descriptivo se utilizaron los siguientes parámetros usando las abreviaturas estándar en inglés (tabla 1): número de vueltas $(\mathrm{WH})$, altura $(\mathrm{SH})$, anchura $(\mathrm{SW})$, altura de la última vuelta (HBW), anchura de la última vuelta (WBW), altura de la apertura $(\mathrm{AH})$ y anchura de la apertura (AW). Estos 7 parámetros de conchas de 20 especímenes de Acatita fueron comparados con los de las poblaciones recientes de 6 localidades en Texas, Estados Unidos y una localidad del río Pánuco, San Luis Potosí, realizadas por Hershler et al. (2011). Para evaluar una posible diferenciación de nuestro material de las poblaciones recientes de Texas y San Luis Potosí los datos morfométricos de las conchas fueron analizados mediante un análisis discriminante. Los 2 ejemplares de Cincinnatia del manantial de Viesca no fueron incluidos en este análisis el cual requiere un mínimo de 15 especímenes. El análisis discriminante fue realizado mediante el programa PAleontogical STatistics v 3.14 (Hammer, Harper y Ryan, 2001). Todo el material fósil, que incluye 153 especímenes, está resguardado en la Colección Malacológica de la 


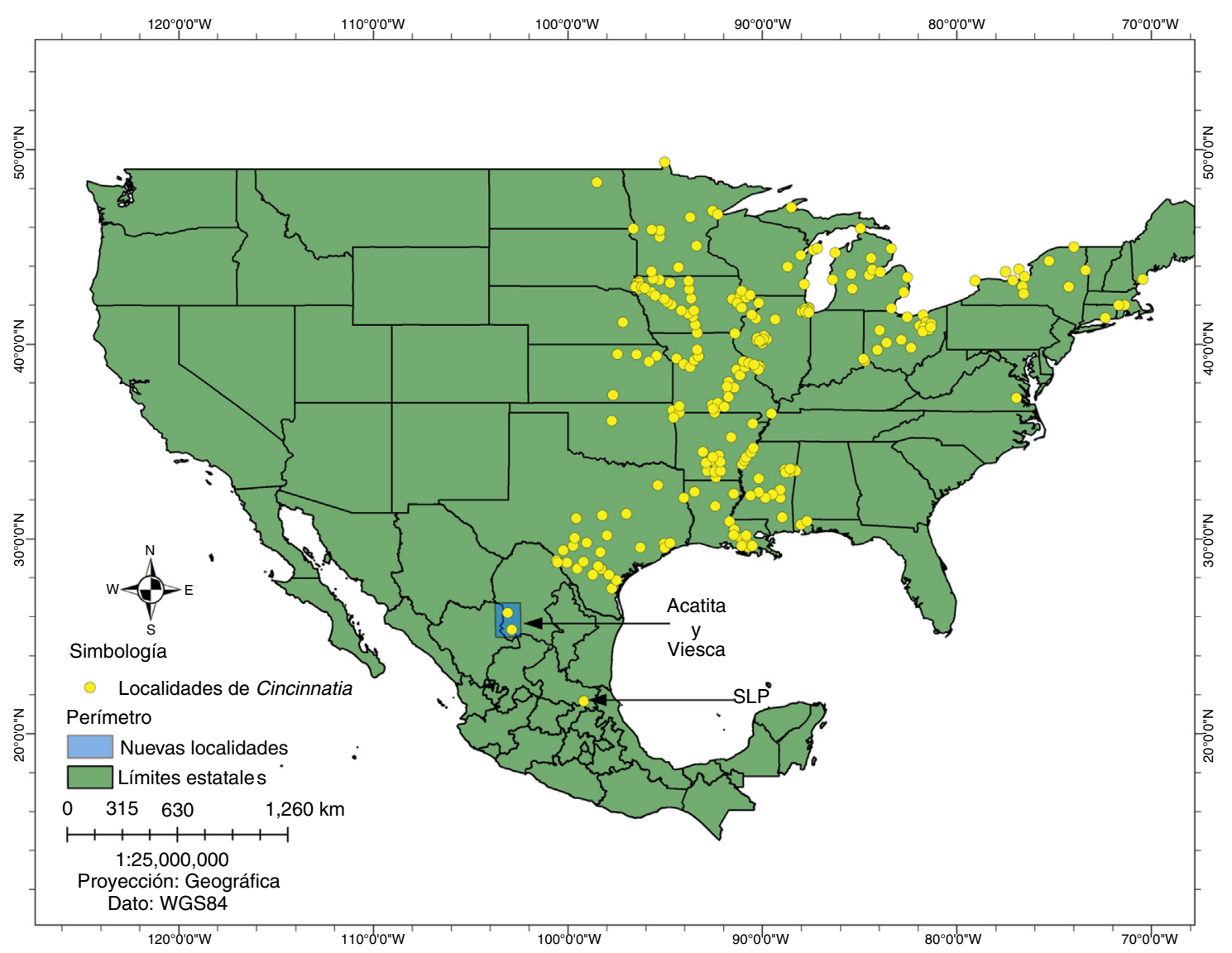

Figura 1. Mapa de la distribución actual de Cincinnatia integra (Say, 1821) con la localización de los nuevos registros (cuadro azul) pleistocenos en el valle de Acatita y subrecientes en Viesca, Coahuila, México (SLP = San Luís Potosí). El color de la figura solo puede apareciarse en la versión electrónica.

Facultad de Ciencias Biológicas de la Universidad Juárez del Estado de Durango, bajo el inventario UJMC-290-320.

\section{Descripción}

Hydrobiidae Troschel, 1857.

Nymphophilinae Taylor, 1966.

Cincinnatia Pilsbry, 1891.

Cincinnatia integra (Say, 1821).

Sinonimia: véase listado en Hershler y Thompson (1996).

\section{Resumen taxonómico}

Material tipo: Paludina integra Say, 1821:174 (perdido, sin topotipos, localidad tipo solo vagamente descrita como «Missouri»). Redescripción realizada por Hershler y Thompson (1996), neotipo USNM 860651, Smithsonian Institution, Washington, EUA.

Localidad tipo (neotipo): río Meramec, $\mathrm{km}$ 2.9 NNO de Steelville, Crawford County, Missouri, EUA.

Material examinado: 151 especímenes (conchas) del valle de Acatita, Coahuila, México (UJCM 290-293, 295-320), recolectados en un corte de aproximadamente $2 \mathrm{~m}$ de espesor de sedimentos fluviátiles compuestos por gravas y arenas $\left(26^{\circ} 16^{\prime} 53.77^{\prime \prime} \mathrm{N}, 103^{\circ} 04^{\prime} 25.96^{\prime \prime} \mathrm{O}\right)$. Leg. A. Czaja, y J.L. Estrada-Rodríguez, 24.01.2012, 16.06.2016. 2 especímenes
(UJCM 294a, 294b) de sedimentos subrecientes de la salida del manantial El Cerrito (2520'23” N, 102 54'29” O), cerca del poblado Viesca, Coahuila, México. Leg. A. Czaja y J.L. Estrada-Rodríguez, 08.05.2016.

Diagnosis (solo conchas): conchas medianas, ovocónicas o subglobosas, con un ápex pronunciado y vueltas redondeadas, apertura grande, redondeada oval, angulada arriba, teloconcha con suturas profundas y un ombligo ampliamente perforado.

Descripción del material del valle de Acatita: concha ovocónica o subglobosa, muy sólida, con una altura entre 4.32$5.45 \mathrm{~mm}$. Ápex ligeramente inclinado, espira con un total de 4.75-5.25 vueltas, un poco menos de 50\% de la altura de la concha, la vuelta de la protoconcha con gránulos finos o lisa en su parte posterior. Las vueltas de la teloconcha aumentan su tamaño regular, con suturas profundas, hombros redondeados y un contorno convexo. Transición protoconcha/teloconcha difícil de precisar, visible solo en forma de aparición de finas estrías de crecimiento. Teloconcha de varios ejemplares con costillas finas espaciadas irregularmente en la penúltima y última vuelta así como más gruesas cerca de la apertura (fig. 2d, e). Abertura redondeada-oval, frecuentemente angulada arriba, con un borde fino y delgado, peristoma completo, labio columelar delgado, arriba ligeramente pegado a la pared de la última vuelta (fig. 2b, $\mathrm{e}, \mathrm{h}$ ), labio exterior un poco más grueso. Ombligo ampliamente perforado (fig. $2 \mathrm{~g}$ ). Conchas de color blanco opaco, opérculo no preservado. Mediciones de las conchas en la tabla 1. 


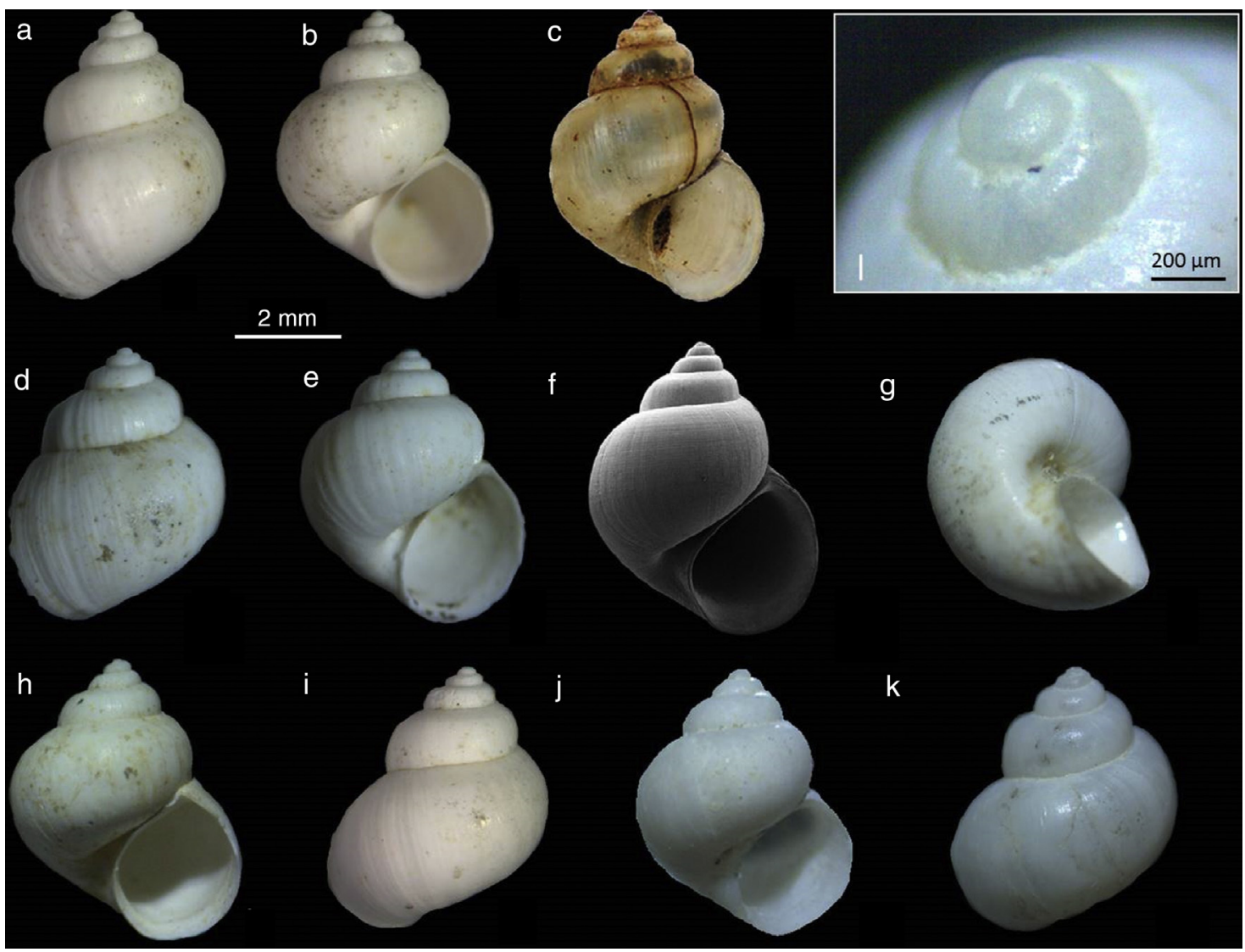

Figura 2. Conchas de Cincinnatia integra (Say, 1821). Material del valle de Acatita, Coahuila, México: a y b) espécimen UJMC-290, vista apertural y abapertural; d y e) espécimen UJMC-291, vista apertural y abapertural, con costillas finas espaciadas irregularmente en la penúltima y última vuelta; g) región umbilical; h e i) 2 especímenes UJMC-292/293, vista apertural y abapertural, con espiras prolongadas. Material de El Cerrito, Viesca, Coahuila, México: j y k) espécimen UJMC-294a, vista apertural y abapertural, 1) protoconcha. Material del Río Meramec, Missouri, EUA: c) espécimen reciente, vista apertural (Hershler et al., 2011, fig. 2b; USNM 860561), con la autorización de los autores). Material de la localidad Veinte de Noviembre, río La Ciénega, San Luis Potosí, México: f) espécimen reciente, vista apertural (Hershler et al., 2011, fig. 3a; UF 283792, con la autorización de los autores).

Descripción del material de El Cerrito, Viesca. Características morfológicas como las descritas del material de Acatita. Abreviaciones véase tabla 1. Espécimen 1: (WH): 4.75; (SH): $4.15 \mathrm{~mm}$; (SW): $3.32 \mathrm{~mm}$; (HBW): $3.88 \mathrm{~mm}$; (WBW): $3.08 \mathrm{~mm}$; $(\mathrm{AH}): 2.24 \mathrm{~mm}$; $(\mathrm{AW}): 1.83 \mathrm{~mm}$. Espécimen 2: (WH): 4.75; (SH): $4.02 \mathrm{~mm}$; (SW): $3.03 \mathrm{~mm}$; (HBW): $3.18 \mathrm{~mm}$; (WBW): $2.62 \mathrm{~mm}$; (AH): $1.99 \mathrm{~mm}$; (AW): $1.75 \mathrm{~mm}$.

Análisis discriminante. Los resultados mostraron que todos los caracteres morfométricos están dentro de la variación fenotípica de la especie reciente, $C$. integra (Say, 1821), lo que justifica la asignación de todo el material fósil del valle de Acatita a esta especie (fig. 3). El análisis indica superposiciones entre las conchas de poblaciones recientes de Texas y San Luis Potosí por un lado y nuestras conchas pleistocenas de Coahuila por otro, y solo pocas diferencias en la morfología (elevado valor de $\mathrm{AH} / \mathrm{SH}$, costillas finas en la penúltima y última vuelta) de las conchas de Acatita. De las 7 poblaciones comparadas, la del valle de Acatita tiene más similitudes morfológicas con conchas de las localidades McMullen y Dimmitt, ambas ubicadas en Texas, EUA.

\section{Discusión}

Las conchas de $C$. integra fueron en el pasado frecuentemente confundidas con conchas de otros miembros de la subfamilia Nymphophilinae con aperturas grandes como Notogillia Pilsbry, 1953, Spilochlamys Thompson, 1968, Floridobia Thompson y Hershler, 2002, Pyrgulopsis Call y Pilsbry, 1886 y Marstonia Baker, 1926. De las 2 especies del género Notogillia, solo $N$. sathon Thompson (1969), posee conchas globosas confundibles con las de Cincinnatia. La diferencia principal es la altura de la apertura en relación con la longitud de la concha $(\mathrm{AH} / \mathrm{SH})$, la cual tiene un valor de 0.60-0.69 para $N$. sathon y de 0.43-0.59 para las poblaciones de Cincinnatia (datos para $N$. sathon según Thompson, 1969). También los valores de SW/SH son diferentes entre ambas especies. Para $N$. sathon el valor es de 0.84-0.96, lo que se refleja en una forma casi circular de la concha, mientras que $C$. integra es más cónica con un valor entre 0.66-0.85. Otra diferencia es que las conchas de $N$. sathon son imperforadas, mientras que las de Cincinnatia son perforadas. 
Tabla 1

Parámetros de las conchas de Cincinnatia integra (Say, 1821) de la población pleistocena del valle de Acatita, Coahuila, en comparación con los de las conchas recientes de poblaciones de Texas, EUA y San Luís Potosí, México (parámetros de las conchas de especies recientes según Hershler et al., 2011).

\begin{tabular}{|c|c|c|c|c|c|c|c|c|c|c|}
\hline & WH & SH & SW & HBW & WBW & $\mathrm{AH}$ & AW & SW/SH & HBW/SH & $\mathrm{AH} / \mathrm{SH}$ \\
\hline \multicolumn{11}{|c|}{ Acatita, Coahuila, México $(n=20)$} \\
\hline Mean & 4.90 & 4.97 & 3.85 & 4.00 & 3.22 & 2.76 & 2.14 & 0.77 & 0.80 & 0.55 \\
\hline SD & 0.23 & 0.29 & 0.20 & 0.23 & 0.20 & 0.16 & 0.14 & 0.68 & 0.79 & 0.55 \\
\hline Range & $4.50-5.25$ & $4.39-5.45$ & $3.29-4.25$ & $3.56-4.46$ & $2.87-3.59$ & $2.41-2.99$ & $1.95-2.39$ & $0.74-0.77$ & $0.65-0.66$ & $0.54-0.55$ \\
\hline \multicolumn{11}{|c|}{ Rio La Ciénega, Veinte de Noviembre, San Luis Potosí, México (n=15) } \\
\hline Mean & 5.15 & 5.36 & 3.90 & 4.22 & 3.17 & 2.83 & 2.24 & 0.73 & 0.79 & 0.53 \\
\hline SD & 0.12 & 0.21 & 0.17 & 0.16 & 0.11 & 0.14 & 0.11 & 0.03 & 0.02 & 0.02 \\
\hline Range & $5.00-5.25$ & $4.98-5.80$ & $3.68-4.31$ & $3.84-4.55$ & $3.01-3.43$ & $2.53-3.15$ & $2.02-2.50$ & $0.67-0.77$ & $0.77-0.81$ & $0.49-0.57$ \\
\hline \multicolumn{11}{|c|}{ Guadalupe River, Comal Co., Texas $(n=20)$} \\
\hline Mean & 5.23 & 4.66 & 3.38 & 3.46 & 2.74 & 2.32 & 1.95 & 0.73 & 0.74 & 0.50 \\
\hline SD & 0.20 & 0.22 & 0.17 & 0.18 & 0.11 & 0.17 & 0.12 & 0.03 & 0.02 & 0.03 \\
\hline Range & $4.75-5.50$ & $4.23-5.12$ & $3.00-3.61$ & $3.14-3.81$ & $2.48-2.95$ & $2.04-2.67$ & $1.72-2.18$ & $0.66-0.77$ & $0.69-0.78$ & $0.43-0.55$ \\
\hline \multicolumn{11}{|c|}{ Nueces River, Dimmit Co., Texas $(\mathrm{n}=27)$} \\
\hline Mean & 5.07 & 4.81 & 3.65 & 3.73 & 2.94 & 2.49 & 2.05 & 0.76 & 0.78 & 0.52 \\
\hline SD & 0.18 & 0.27 & 0.17 & 0.19 & 0.13 & 0.11 & 0.11 & 0.03 & 0.02 & 0.02 \\
\hline Range & $4.75-5.25$ & $4.32-5.63$ & $3.42-4.12$ & $3.44-4.17$ & $2.72-3.29$ & $2.29-2.70$ & $1.90-2.33$ & $0.70-0.82$ & $0.74-0.81$ & $0.47-0.56$ \\
\hline \multicolumn{11}{|c|}{ Las Moras Creek, Brackettville, Kinney Co., Texas, $(n=15)$} \\
\hline Mean & 5.57 & 6.58 & 4.84 & 4.95 & 3.84 & 3.23 & 2.75 & 0.74 & 0.75 & 0.49 \\
\hline SD & 0.26 & 0.65 & 0.47 & 0.38 & 0.34 & 0.18 & 0.22 & 0.04 & 0.03 & 0.03 \\
\hline Range & $5.00-6.00$ & $5.16-7.59$ & $4.13-5.56$ & $4.20-5.50$ & $3.31-4.47$ & $2.94-3.54$ & $2.40-3.03$ & $0.68-0.85$ & $0.71-0.81$ & $0.45-0.59$ \\
\hline \multicolumn{11}{|c|}{64 km NE de Houston, carratera HWY 90, Liberty Co., Texas $(n=19)$} \\
\hline Mean & 5.25 & 5.24 & 3.80 & 3.99 & 3.09 & 2.70 & 2.15 & 0.73 & 0.76 & 0.52 \\
\hline SD & 0.24 & 0.40 & 0.24 & 0.27 & 0.19 & 0.18 & 0.14 & 0.03 & 0.02 & 0.03 \\
\hline Range & $4.75-5.75$ & $4.60-6.03$ & $3.44-4.26$ & $3.52-4.51$ & $2.78-3.42$ & $2.31-2.97$ & $1.91-2.40$ & $0.68-0.79$ & $0.71-0.81$ & $0.48-0.57$ \\
\hline \multicolumn{11}{|c|}{ Frio River, McMullen Co., Texas (n=20) } \\
\hline Mean & 5.09 & 4.82 & 3.62 & 3.78 & 2.91 & 2.51 & 2.05 & 0.75 & 0.79 & 0.52 \\
\hline SD & 0.20 & 0.38 & 0.21 & 0.27 & 0.19 & 0.18 & 0.13 & 0.03 & 0.02 & 0.02 \\
\hline Range & $4.75-5.25$ & $4.31-5.67$ & $3.25-4.18$ & $3.36-4.30$ & $2.59-3.39$ & $2.30-2.87$ & $1.81-2.27$ & $0.71-0.80$ & $0.76-0.81$ & $0.48-0.56$ \\
\hline
\end{tabular}

AH (aperture height): altura de la apertura (mm); AW (aperture width): anchura de la apertura (mm); HBW (height of body whorl): altura de la última vuelta (mm); SH (shell height): altura de la concha (mm); SW (shell width): anchura de la concha (mm); WBW (width of body whorl): anchura de la última vuelta (mm); WH (total shell whorls): número total de las vueltas.

De las 3 especies del género Spilochlamys solo Spilochlamys turgida Thompson, 1969, presenta cierto parecido en sus conchas con las de Cincinnatia. Pero ya Thompson (2004), autor del género, aclara que $S$. turgida difiere de $C$. integra: «...by having a more deeply impressed suture, more strongly shouldered whorls, and a wider umbilical perforation». Pero la diferencia principal es la espira fuertemente aplastada de las conchas de S. turgida, una característica que no se observa en C. integra.

La mayoría de las 14 especies del género Floridobia tiene conchas pequeñas $(2-4 \mathrm{~mm})$ con una espira muy prolongada y una forma cónica. Casi todas las especies de este género, originalmente descritas como Cincinnatia, son endémicas y restringidas frecuentemente a un solo manantial o lago en EUA (Thompson, 2004). La comparación señala que conchas de Floridobia se parecen más a las del género Pyrgulopsis. Esta semejanza conchológica refleja bien la cercanía filogenética de ambos géneros comprobada por Liu y Hershler (2005) y subraya otra vez la (frecuentemente negada) utilidad de la morfología de las conchas para fines taxonómicos. La mayoría de las 141 especies de Pyrgulopsis tienen conchas mucho más pequeñas (entre $2-4 \mathrm{~mm}$ ) y con espiras muy prolongadas. Las pocas especies

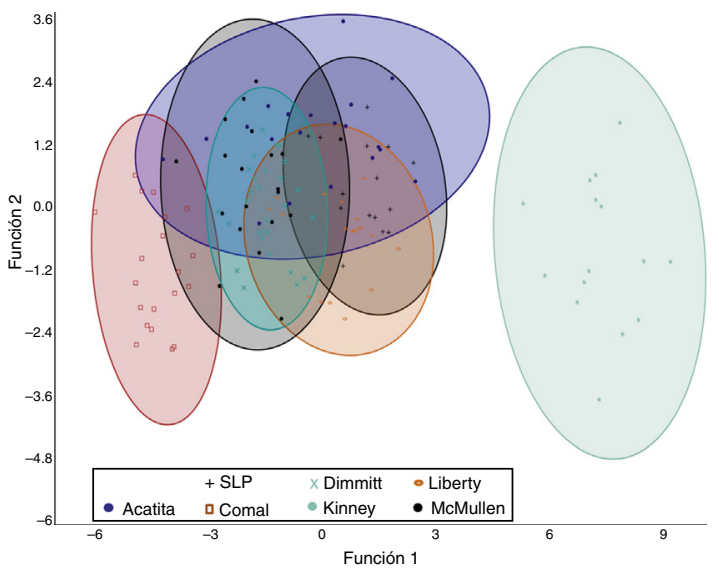

Figura 3. Gráfica de las 2 primeras funciones discriminantes (señalando $91.84 \%$ y $5.03 \%$ de la variación total) basada en 7 parámetros de las conchas de 7 poblaciones de Cincinnatia integra (tabla 1). Las elipses demuestran una diferenciación limitada del material pleistoceno del valle de Acatita, Coahuila con respecto a las poblaciones recientes de Texas y San Luís Potosí (abreviaciones de las localidades véase tabla 1 ). 
de Pyrgulopsis como Pyrgulopsis intermedia (Tryon, 1865), Pyrgulopsis idahoensis (Pilsbry, 1933), Pyrgulopsis hendersoni (Pilsbry, 1933), Pyrgulopsis nevadensis (Stearns, 1833), Pyrgulopsis robusta (Walker, 1908) y otras que alcanzan el tamaño de Cincinnatia, tienen otra forma general de la concha (más cónica con una espira prolongada) lo que las diferencia claramente de este género.

Especialmente una especie del género Marstonia, Marstonia comalensis (Pilsbry y Ferriss, 1906), fue en el pasado posiblemente la más confundida con $C$. integra. Pero ya Hershler y Lui (2011) mencionan que las 2 especies difieren tanto anatómicamente como también en la morfología de sus conchas. Las diferencias principales son el considerable tamaño menor (3.20$3.87 \mathrm{~mm}$ ), una espira más prolongada, así como la protoconcha casi plana de conchas de $M$. comalensis. Además, las conchas de Cincinnatia poseen aperturas más largas con respecto a su tamaño representado por el valor de $\mathrm{AH} / \mathrm{SH}$ de 0.51 , comparado con el de Marstonia de 0.45 .

\section{Agradecimientos}

Los autores agradecen al Dr. Robert Hershler, Smithsonian Institution, Washington, DC, EUA, sus importantes impulsos para realizar el presente trabajo. También al Dr. Alejandro M. Maeda Martínez, por sus sugerencias y correcciones al manuscrito.

\section{Referencias}

Butzer, K. W., Abbott, J. T., Frederick, C. D., Lehman, P. H., Cordova, C. E. y Oswald, J. F. (2008). Soil-geomorphology and «wet» cycles in the Holocene record of North-Central Mexico. Geomorphology, 101, 237-267.

Czaja, A. y Estrada-Rodríguez, J. L. (2015). Two new species of Tryonia (Caenogastropoda: Cochliopidae) from the Late Pleistocene of Coahuila, Northern Mexico. The Nautilus, 129, 83-89.
Czaja, A., Estrada-Rodríguez, J. L. y Romero-Méndez, U. (2014). Freshwater mollusks of the Valley of Sobaco, Coahuila, Northeastern Mexico -a subfossil ecosystem similar to Cuatrociénegas. Boletín de la Sociedad Geológica Mexicana, 66, 459-469.

Czaja, A., Estrada-Rodríguez, J. L. y Romero-Méndez, U. (2015). A new species of the genus Mexipyrgus Taylor, 1966 (Caenogastropoda: Truncatelloidea: Cochliopidae) from late Holocene spring deposits in Viesca, Coahuila, Mexico. The Nautilus, 129, 163-168.

Hammer, ̌̌., Harper, D. A. T. y Ryan, P. D. (2001). PAST: Paleontological statistics software package for education and data analysi. [consultado 8 Dic 2016]. Disponible en: http://palaeo-electronica.org/2001_1/past/issue1_01.htm

Hershler, R. y Liu, H. P. (2011). Redescription of Marstonia comalensis (Pilsbry \& Ferriss, 1906), a poorly known and possibly threatened freshwater gastropod from the Edwards Plateau region (Texas). ZooKeys, 77, 1-16.

Hershler, R. y Thompson, F. G. (1996). Redescription of Paludina integra Say, 1821, type species of genus Cincinnatia (Gastropoda: Hydrobiidae). Journal of Molluscan Studies, 62, 33-55.

Hershler, R., Thompson, F. G. y Liu, H. P. (2011). A large range extension and molecular phylogenetic analysis of the monotypic North American aquatic gastropod genus Cincinnatia (Hydrobiidae). Journal of Molluscan Studies, 77, 232-240.

La Rocque, A. (1970). Pleistocene Mollusca of Ohio. Ohio Geological Survey Bulletin, 62, 1-553.

Liu, H. P. y Hershler, R. (2005). Molecular systematics and radiation of western North American nymphophiline gastropods. Molecular Phylogenetics and Evolution, 34, 284-298.

Oseguera, J. R. (2012). Ostracodes and gastropods of the Waucobi Lake Beds: significance for Pliocene environmental and climate conditions in the Eastern Sierra Nevada, California (Tesis de maestría no publicada) [consultado 8 Dic 2016]. Disponible en: http://escholarship.org/uc/item/5m33c5x5. Riverside: University of California.

Pilsbry, H. A. (1939). Land Mollusca of North America North of Mexico vol. I part 1. Philadelphia: Academy of Natural Sciences of Philadelphia.

Thompson, F. G. (1969). Some hydrobiid snails from Georgia and Florida. Quarterly Journal of the Florida Academy of Sciences, 32, 241-265.

Thompson, F. G. (2004). An identification manual for the freshwater snails of Florida [consultado 8 Dic 2016]. Disponible en: http://www.flmnh.ufl.edu/malacology/fl-snail/snails1.htm 